\title{
Caracterização e Modelagem Temporal de Chamadas de Telefonia Móvel em Grandes Eventos
}

\author{
Lucas Maia Silveira', Humberto T. Marques-Neto ${ }^{1}$ \\ ${ }^{1}$ Departamento de Ciência da Computação \\ Pontifícia Universidade Católica de Minas Gerais (PUC Minas) \\ 30.535-901 - Belo Horizonte - MG - Brasil \\ lmsilveira@sga.pucminas.br, humberto@pucminas.br
}

\begin{abstract}
The increasing use of mobile phones challenges operators to evolve and improve their services based on the behavior of their users. In particular, large-scale events may impose standards that differ strongly from those observed in normal daily use of the mobile networks. This paper presents the impact of football matches on the workload of a mobile network based on the use of a proposed methodology. The results show the dynamics of the workload surrounding the times of these events featuring the increased use of voice services due to the displacement of both participants and the nature of the event itself.
\end{abstract}

Resumo. O uso cada vez maior de telefones celulares desafia as operadoras a evoluírem e melhorarem os seus serviços com base no comportamento de seus usuários. Em particular, eventos de grande escala podem impor padrões que diferem fortemente daqueles observados normalmente no uso diário das redes das operadoras de telefonia celular. Este trabalho apresenta os impactos de jogos de futebol na carga de trabalho de uma rede de telefonia móvel a partir do uso de uma metodologia proposta. Os resultados mostram a dinâmica da carga de trabalho no entorno do horário dos eventos e apresentam características do aumento de uso dos serviços de voz da operadora em consequência tanto do deslocamento dos participantes quanto da natureza do próprio evento.

\section{INTRODUÇÃO}

Analisar os padrões de mobilidade humana de usuários da telefonia móvel é um grande desafio. Contudo, também pode ser uma grande oportunidade para se entender melhor as características da mobilidade humanas em uma determinada área geográfica. Este entendimento pode ajudar os governos e as empresas a preverem e planejarem ações proativas para melhorar a qualidade de vida nas cidades [González; Hidalgo; Barabási, 2008; Soper, 2012]. Tais previsões e planejamento de ações podem ser feitos a partir de modelos e padrões de mobilidade construídos a partir de grandes conjuntos de dados anônimos de uso da rede de telefonia móvel [Candia et al., 2008].

Embora estudos recentes mostrem que a mobilidade humana em áreas urbanas pode ser bem previsível com base em rotinas diárias [Song et al., 2010b], as operadoras de telefonia celular ainda têm dificuldades para planeja a infraestrutura de comunicação necessária, capaz de suportar as cargas de trabalho incomuns que, normalmente são 
percebidas durante eventos de grande escala [Bleicher,2012]. Mesmo sabendo que muitos desses eventos de grande porte são programados e planejados com antecedência, e que provavelmente causarão mudanças na carga de trabalho [Bagrow; Wang; Barabási,2011], ainda é comum perceber o congestionamento de recursos das redes de telefonia celular das operadoras durante esses eventos de grande porte.

Neste trabalho, o aluno de iniciação científica, Lucas Maia Silveira, aplicou a metodologia proposta em Xavier (2012), trabalho também de sua autoria, para geração dos mapas de calor com o propósito de representar a mobilidade humana durante eventos de grande escala. Os estudos foram baseados em chamadas móveis feitas ao longo das principais vias rodoviárias para o Estádio Engenhão, no Rio de Janeiro, Brasil, que hospedou partidas do Campeonato Brasileiro de Futebol em 2011. Por fim, este trabalho apresenta a caracterização de chamadas de celular realizadas na infraestrutura de suporte antes, durante e depois de duas partidas de futebol entre as mesmas equipes (Flamengo e Vasco), que aconteceram em Agosto e Dezembro de 2011, bem como a visualização dos resultados em mapas de calor que podem representar a mobilidade humana nesses períodos de tempo.

No geral, os resultados apontam que a ocorrência de um evento de grande escala modifica o volume de trabalho típico da rede de telefonia móvel. Eles também sugerem que, embora atípica, essa dinâmica de carga de trabalho segue um padrão, motivando a futura concepção de aplicações-alvo e dispositivos para eventos dessa natureza.

O restante deste trabalho está organizado em 4 capítulos. O capítulo 2 contém outros trabalhos que abordam tema similar. No capítulo 3 serão explicitadas as etapas da metodologia utilizada no projeto. Na quarta seção os resultados obtidos serão apresentados em mapas de calor e discutidos. Por último, a conclusão sobre as respostas obtidas.

\section{RERENCIAL TEÓRICO}

O crescimento e a prevalência atual de redes de telefonia móvel na sociedade contemporânea requerem estudos para melhor compreender a sua utilização [Eagle; Pentland; Lazer, 2009; Simonite, 2010]. As operadoras de telefonia celular costumam manter registros de chamadas de todos os usuários, incluindo a localização aproximada (com base em suas antenas) em que cada chamada começou e terminou. [Song et al., 2010b]. Esses conjuntos de dados anônimos podem ser usados para se analisar o comportamento dos usuários de telefones celulares em diferentes períodos do dia [Candia et al., 2008].

González, Hidalgo e Barabási (2008) apontam que é possível prever os padrões de mobilidade dos usuários, apesar do comportamento ser diferente de cada pessoa em relação ao seu trabalho, família, preferências de lazer, e assim por diante. Essa observação sugere que os padrões de mobilidade podem ser uma valiosa contribuição para conduzir o planejamento urbano de uma cidade, por exemplo, para melhorar o tráfego de veículos, para apoiar a tomada de decisões e ações em situações de emergência, e até mesmo para melhorar o controle da disseminação de uma doença em uma região [Balcan et al., 2009]. Em geral, os padrões de comportamento dos usuários de telefone celular podem ser modelados através de mineração de dados coletados em infraestrutura das operadoras [González; Barabási, 2007; Mitchell,2009]. Isso pode permitir a realização de um planejamento urbano para melhor responder a emergências 
e acarretarem um melhor fluxo de veículos no trânsito. Song et al. (2010c) também utilizam um modelo para tentar identificar um padrão na mobilidade humana, apesar do ser humano apresentar uma trajetória aleatória durante o dia.

Esses estudos são importantes, pois além de prever o fluxo do trânsito ajudam no planejamento urbano, na saúde pública e nos aspectos econômicos. Assim, é possível conhecer quais regiões são mais atraentes para investimentos em lojas, indústrias, entre outros. Além disso, no caso da saúde pública, é possível prevenir epidemias, já que de posse do conhecimento da movimentação na cidade é possível medicar primeiramente as áreas criticas, além de prever como será o fluxo das pessoas caso ocorra alguma emergência (furacões, terremotos, etc).

Percebe-se no trabalho de Bagrow, Wang e Barabási (2011) que quando ocorre algum tipo de emergência, o padrão de atividade humana se altera. Além disso, há um aumento quase instantâneo na utilização da rede móvel nas proximidades do local do evento. Com essa grande quantidade de dados que inunda a rede repentinamente e permanece alta por um período de tempo considerável, é possível identificar e classificar aquele evento como uma emergência. Quando se trata de shows, partidas de futebol e outras atividades de lazer, a quantidade de ligações aumenta gradualmente no início, diminui um pouco durante e torna a aumentar na saída do local por um período curto após o qual cai para quase zero a utilização da rede móvel [Xavier et al.,2012]

Em Song et al. (2010b), os autores utilizam um modelo para identificar padrões de mobilidade e prever o comportamento humano em comum (ou seja, típico), bem como em situações anormais e inesperadas. Durante os eventos inesperados, diferentes padrões de mobilidade são observados. Esses eventos causam aumento no uso de redes de telefonia móvel próximas ao local. Bagrow, Wang e Barabási (2011) argumentam que as características da carga de trabalho de rede podem indicar o tipo de evento que os usuários estão experimentando, por exemplo, uma emergência, um evento de esporte ou um concerto.

Os dados de mobilidade são gerados quando um usuário utiliza recursos das antenas responsáveis por prover os serviços de telefonia móvel através de seus aparelhos móveis. Song et al. (2010b) falam sobre como coletar esses dados.. A partir daí é possível calcular a quantidade de ligações em determinada hora/local e prever como será o comportamento das pessoas durante o dia. Isso pode auxiliar no planejamento urbano, no bem estar e na saúde pública.

\section{METODOLOGIA}

Para alcançar o objetivo de caracterizar e modelar o uso dos serviços de rede móvel, foram utilizadas bases de dados reais de 2011, com o propósito de auxiliar no planejamento da capacidade, no gerenciamento da infraestrutura tecnológica e no posicionamento geográfico dos pontos de conexão (antenas) da rede de telefonia móvel. Essas bases também foram utilizadas para os modelos que representam o padrão de mobilidade humana em dias com eventos (ex.: eventos esportivos) e em dias normais. A seguir será detalhada cada etapa da metodologia de caracterização.

\subsection{Caracterização e Modelagem da Base de Dados}

A proposta de caracterização consiste em identificar quem estava no evento, de onde esses participantes vieram e que rotas foram utilizadas por eles na saída. Isso é 
realizado através da quantidade de ligações de celulares feitas nas antenas que se localizam nas proximidades do local do evento durante sua realização, com foco no grupo que estava envolvido diretamente (quem foi participar/assistir) e indiretamente (vendedores e pessoas que passam pelas proximidades desse local).

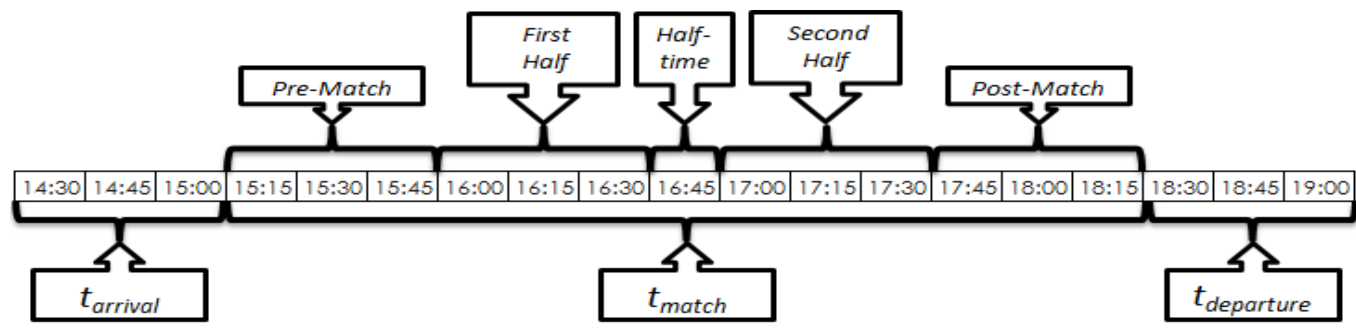

Figura 1. Linha do Tempo para partidas de futebol

A fim de realizar a análise com base no que foi dito acima, utilizou-se uma linha do tempo específica para jogos de futebol [Xavier et al., 2012]. A Figura 1 apresenta essa linha do tempo, que representa o tempo analisado durante os dias com jogos no Engenhão, realizados no intervalo de $16 \mathrm{~h}$ até 17:45. O intervalo de 45 minutos considerado antes (Pre-Match) e depois (Post-Match) foi definido com o objetivo de identificar os horários de chegada e saída dos torcedores ou envolvidos no jogo. O $t_{\text {arrival }}$ e o $t_{\text {departure }}$ auxiliam na identificação das rotas utilizadas pelos usuários da rede de telefonia celular que realizam chamadas no $t_{\text {match }}$.

Nos dias 30/10/2011 e 04/12/2011, as análises foram feitas adiantando o horário em uma hora, ou seja, $16 \mathrm{~h}$ da linha do tempo correspondem às $17 \mathrm{~h}$ nesses dois dias. Isto foi realizado por causa do horário de verão que se iniciou no dia 21/10/2011.

\subsection{Geração dos Mapas de Calor}

Para estudar o dinamismo da carga de trabalho a partir do local do evento, foi desenvolvida pelo aluno de iniciação científica, autor desse artigo, uma forma de utilizar mapas de calor para representar a intensidade de utilização nas antenas pelos usuários do serviço de telefonia móvel.

A primeira versão proposta pelo aluno foi baseada no-uso da sobreposição de polígonos no mapa. Esses polígonos, no caso, círculos, foram criados utilizando a API do Google Maps Javascript antes da atualização da versão 27/07/2012. Nesta versão, os dados eram inseridos manualmente no arquivo para gerar um mapa por vez. Com isso, a geração dos mapas era demorada. Após a atualização de 27/07 a API passou a ter um novo tipo de camada: os mapas de calor. Com isso, a representação de círculos foi trocada para uma representação de mapas térmicos de melhor qualidade de visualização. Contudo, os dados ainda continuavam sendo mapeados com base numa representação de cor nas respectivas localizações das antenas na região.

Após a implementação dos mapas de calor, foi necessário automatizar o processo de geração. Para isso construiu-se uma função em $\mathrm{C}++$ que recebia como entrada, dentro de um determinado intervalo de tempo, as coordenadas geográficas das antenas (latitude e longitude) e a quantidade de ligações, criando assim o arquivo html, a partir do qual o mapa era gerado. Assim, a geração tornou-se bem mais eficiente, sendo possível fazer mais de um mapa simultaneamente. Com essa automatização, também foi possível gerar vídeos que mostram o transcorrer da linha do tempo do 
evento. Nesses vídeos, cada frame corresponde a um mapa de um determinado intervalo de tempo.

\section{RESULTADOS}

Esta seção descreve as bases de dados utilizadas e mostra o uso dos mapas de calor para representação da mobilidade humana nos eventos analisados, ou seja, nos jogos de futebol realizados no Engenhão em 2011.

\subsection{Descrição da Base de Dados}

Após a definição da linha do tempo de observação (conforme Figura 1), é necessário identificar as antenas que cobrem a região do evento. Para o caso dos jogos, foram identificadas quatro antenas, localizadas em um raio de 1.500 metros do Engenhão. Depois disso, deve-se selecionar os usuários que fizeram ligação nessas antenas do intervalo do evento $\left(t_{\text {match }}\right.$ e $t_{\text {event }}$ ), que vai do início desse intervalo (PreMatch e Pre-Event) até o final (Post-Match e Post-Event). Essa seleção tem como objetivo identificar quem participou do evento para podermos realizar uma análise desses usuários da rede de telefonia celular.

Com a identificação de quem estava no evento, é possível analisar o seu comportamento durante dois outros intervalos, o $t_{\text {arrival }}$ e o $t_{\text {departure }}$, os quais mostram como foi a chegada (de onde vieram) e como foi a partida (para onde foram) daqueles que estiveram na região do evento.

A Tabela 1 apresenta a quantidade de ligações e usuários que cada base de dados possui. Nessa tabela é mostrada a quantidade de chamadas realizadas no intervalo de 14h15min até 19h15min (período analisado) e também a quantidade de usuários distintos identificados no mesmo intervalo de tempo. Nessa tabela, são apresentadas as ligações realizadas pelos torcedores e o total de chamadas realizada para cada dia. Além disso, foram identificadas todas as pessoas que fizeram ligações e quais delas eram os espectadores (aqueles que realizaram chamadas nas antenas próximas ao evento). Podese perceber que, nos dias de jogo (28/08 e 04/12), a quantidade de ligações é superior ao dia sem jogo (30/10). Outro fato interessante é que o dia 04/12, apesar de haver menos ligações totais, há mais ligações em torno do Engenhão do que no dia 28/08. Isso se deve ao fato de aquele ser o jogo da rodada final, consequentemente atraindo mais torcedores do que partidas de rodadas anteriores.

Tabela 1. Visão geral do Engenhão

\begin{tabular}{|c|c|c|c|c|}
\hline Dias & Chamadas & Chamadas Espectadores & Usuários & Espectadores \\
\hline 28 de Agosto de 2011 & 50138 & 3362 & 28921 & 1366 \\
\hline 04 de Dezembro de 2011 & 47776 & 4284 & 27290 & 1754 \\
\hline 30 de Outubro de 2011 & 41021 & 1270 & 25313 & 691 \\
\hline
\end{tabular}

\subsection{Mapas de Calor}

As Figuras 2, 3 e 4 representam, respectivamente, os mapas $t_{\text {arrival }}$, $t_{\text {match }} \mathrm{e}$

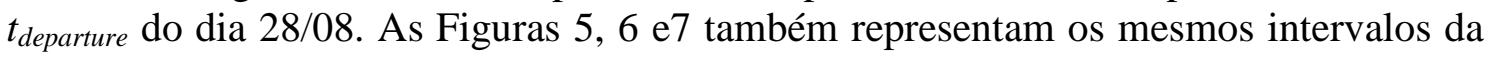


linha do tempo ( $t_{\text {arrival }}, t_{\text {match }}$ e $t_{\text {departure }}$ ) para o jogo da rodada final (dia 04/12). Por último, as Figuras 8, 9 e 10 mostram os mesmos períodos para o dia sem jogo (30/10). Nos mapas dos dias do jogo, se pode perceber uma quantidade maior de áreas avermelhadas do que no dia sem jogo. Portanto, os dias de eventos realmente causam impacto na região.

Outro fator interessante é a possibilidade de identificar três rotas para se chegar ao estádio, uma vindo pela Avenida ao Sul (parte de baixo do mapa) e duas vindo pelo Leste. Dessas duas, uma é outra avenida que chega por cima no Engenhão e a outra é a linha de metrô. Nessa última rota percebe-se uma concentração maior de pessoas. Portanto, pode se concluir que este transporte público é mais utilizado pelos espectadores do que automóveis para ir à partida.

Por último, é possível perceber que no mapa da Figura 3 a quantidade de ligações é muito grande, levando em consideração o mesmo intervalo de tempo do dia 04/12 (Figura 6). Isso se deve ao fato do técnico do Vasco ter sofrido um acidente vascular cerebral, o que pode ter gerado o pico de ligações no intervalo de análise. Isso, difere do padrão encontrado no dia 04/12, que possui mais ligações antes (Figura 5) e depois (Figura 7) do jogo.

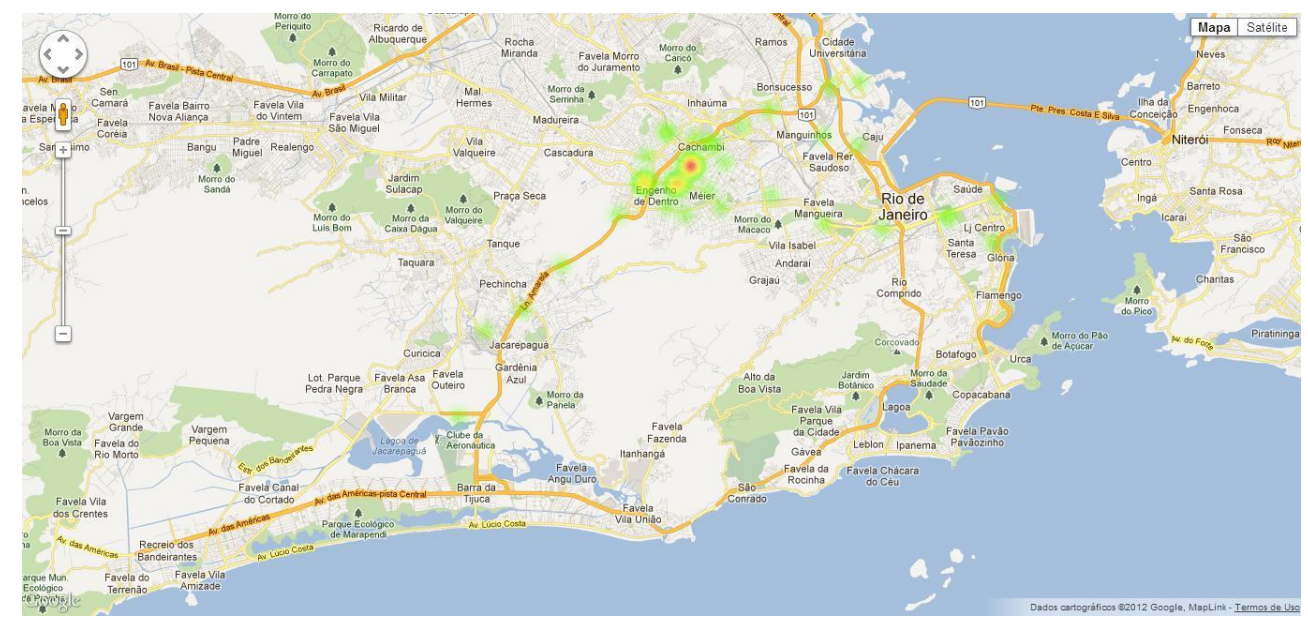

Figura 2. Mapa dia 28/08/11 - $t_{\text {arrival }}$

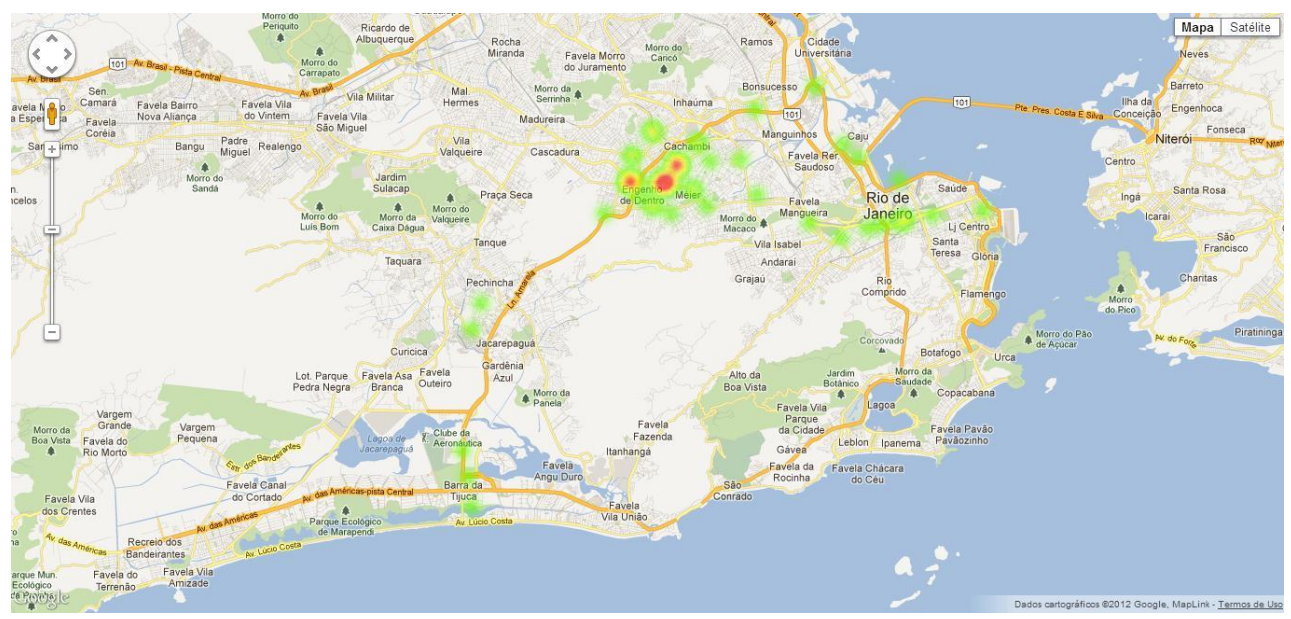

Figura 3. Mapa dia 28/08/11 $-t_{\text {match }}$ 


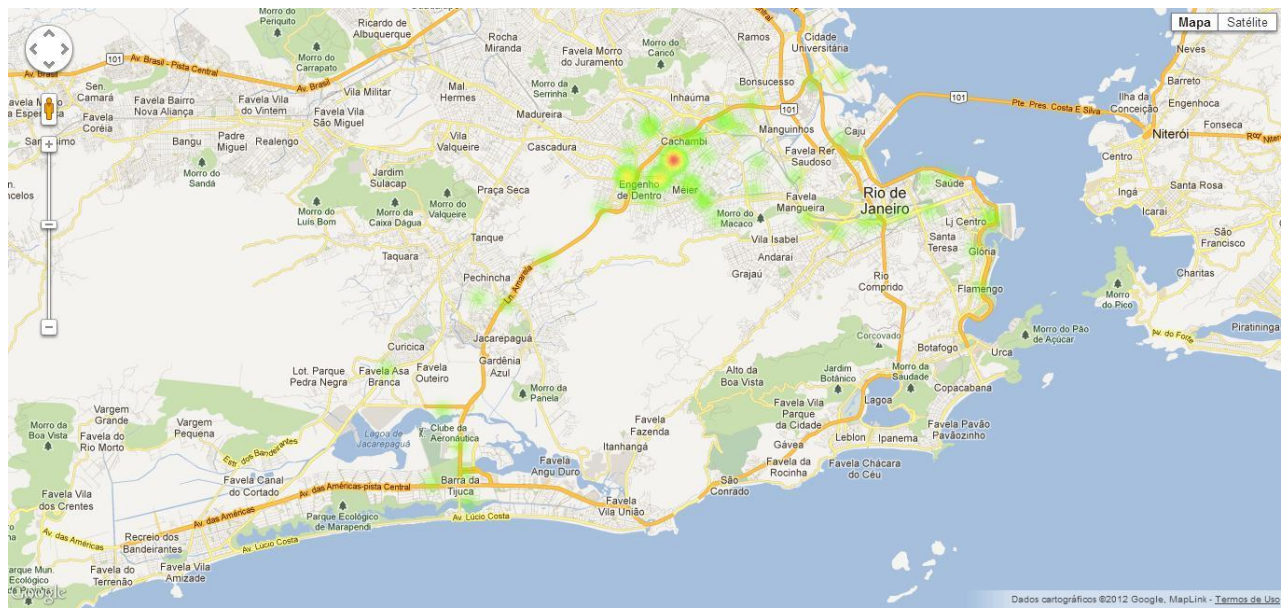

Figura 4. Jogo dia 28/08/11 $-t_{\text {departure }}$

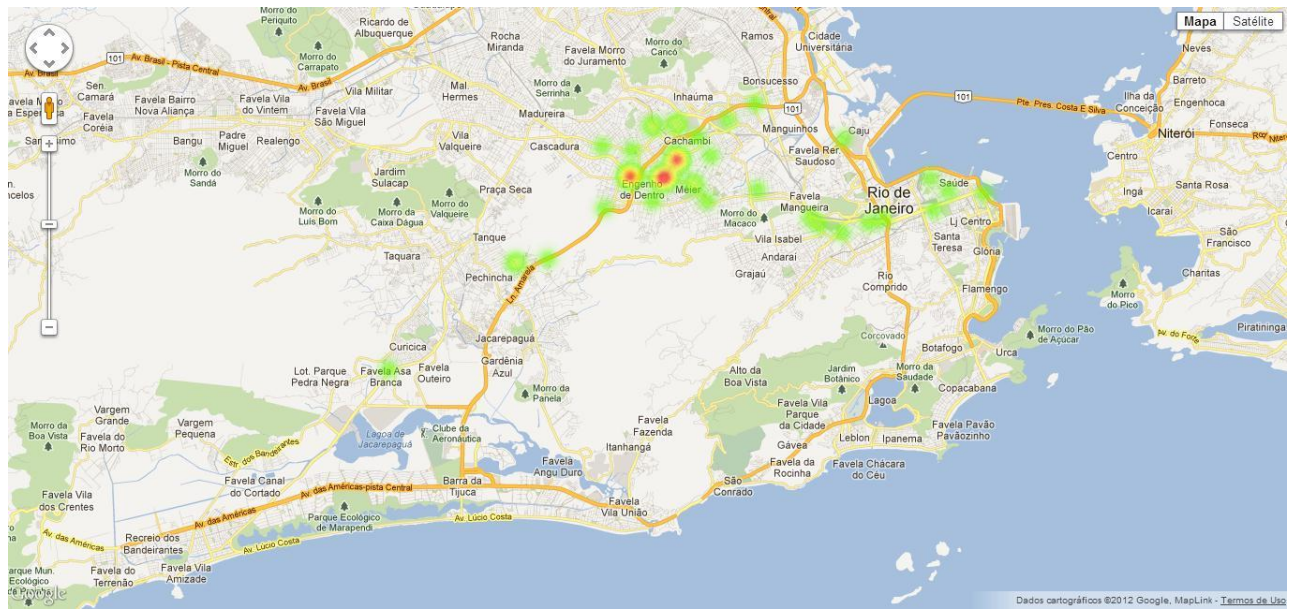

Figura 5. Mapa dia 04/12/11 $-t_{\text {arrival }}$

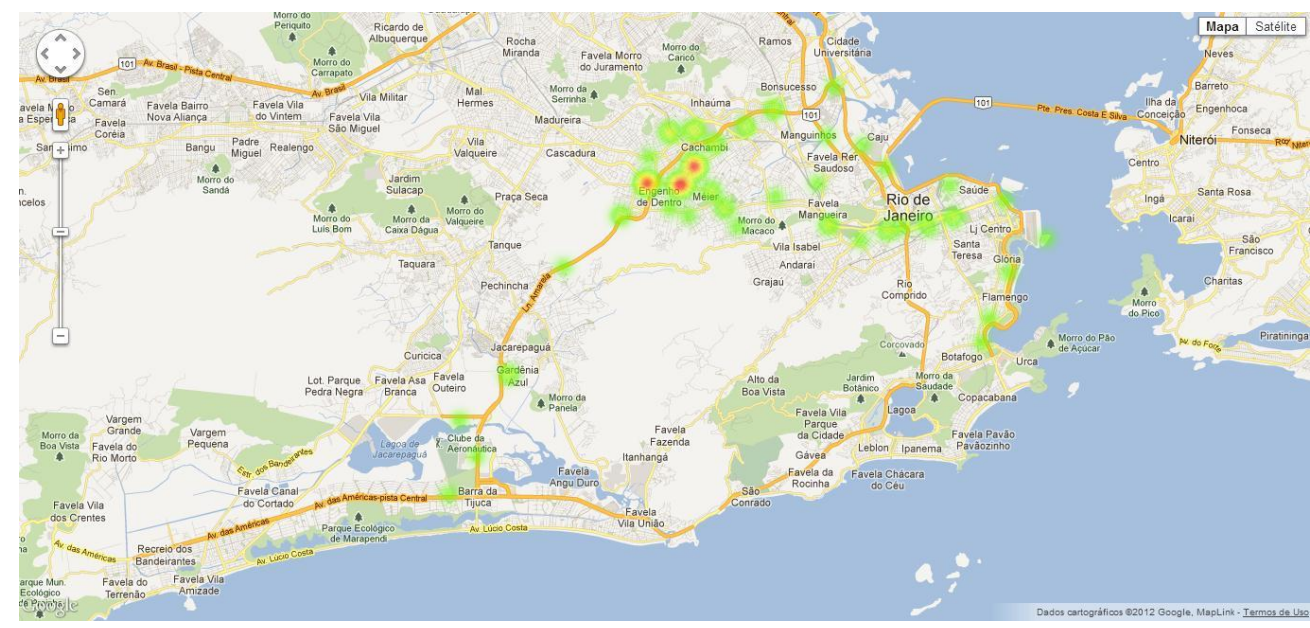

Figura 6. Mapa dia 04/12/11 $-t_{\text {match }}$ 


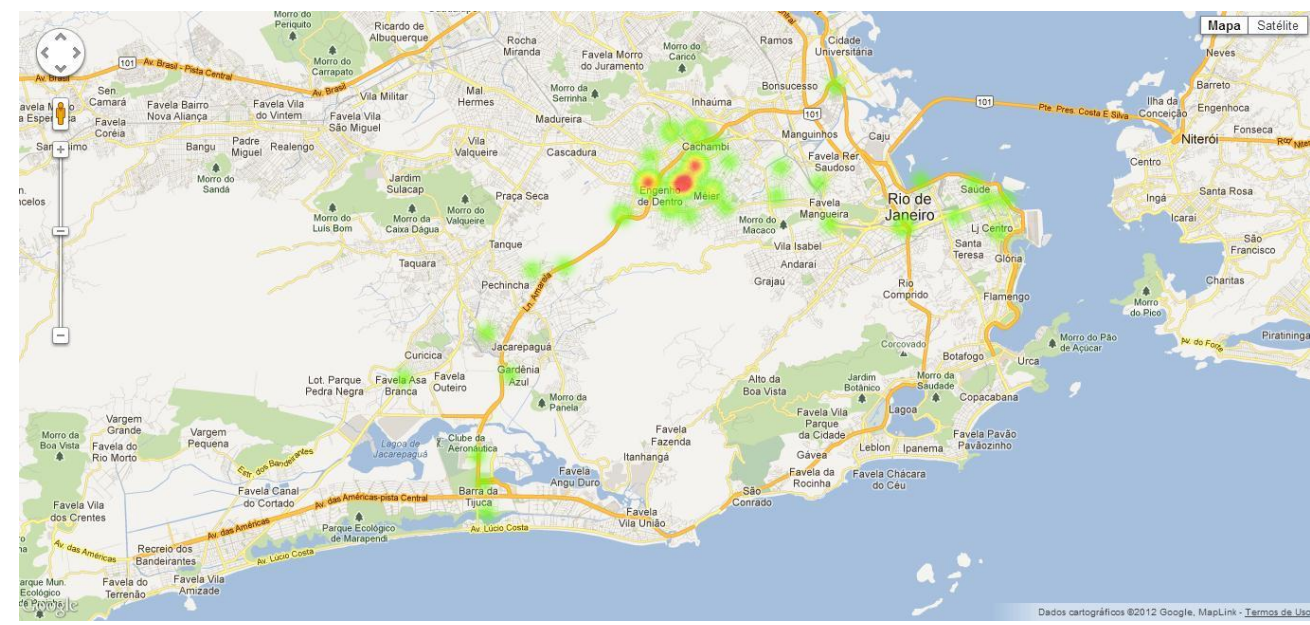

Figura 7. Mapa dia 04/12/11 $-t_{\text {departure }}$

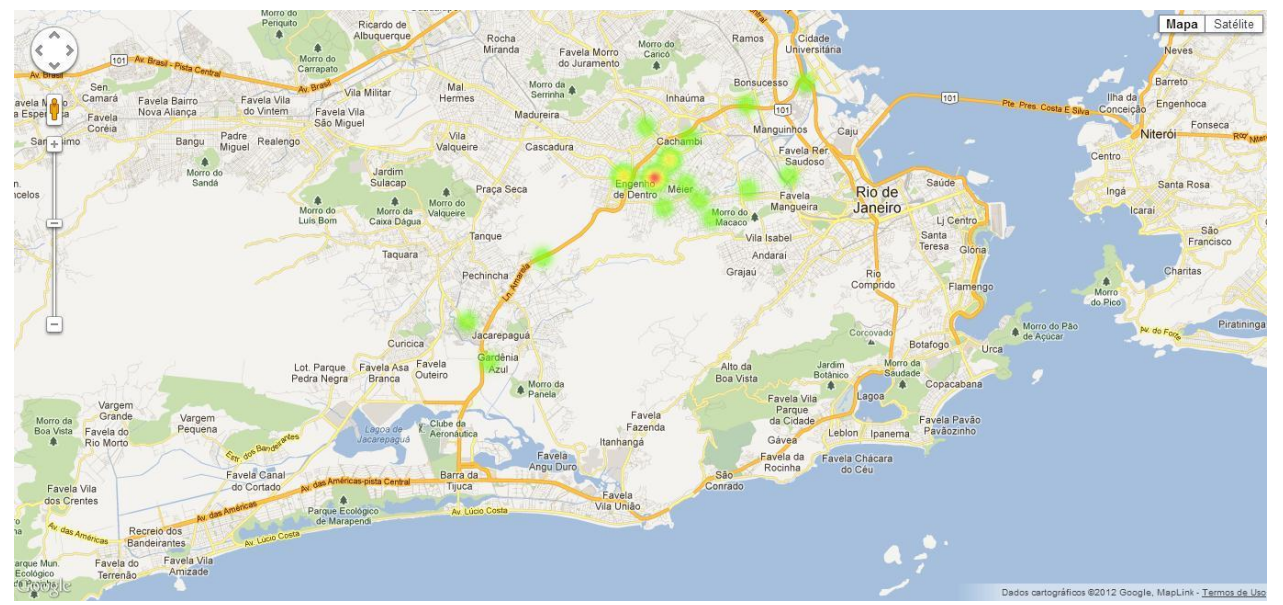

Figura 8. Mapa dia 30/10/11 $-t_{\text {arrival }}$

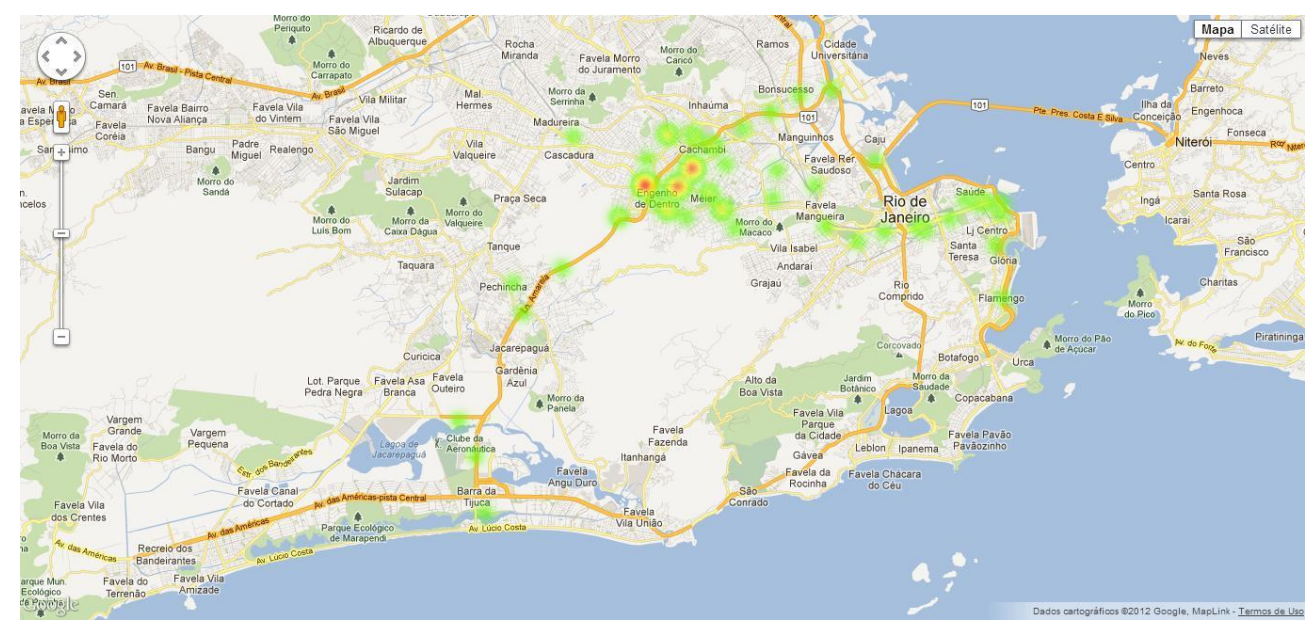

Figura 9. Mapa dia 30/10/11 $-t_{\text {match }}$ 


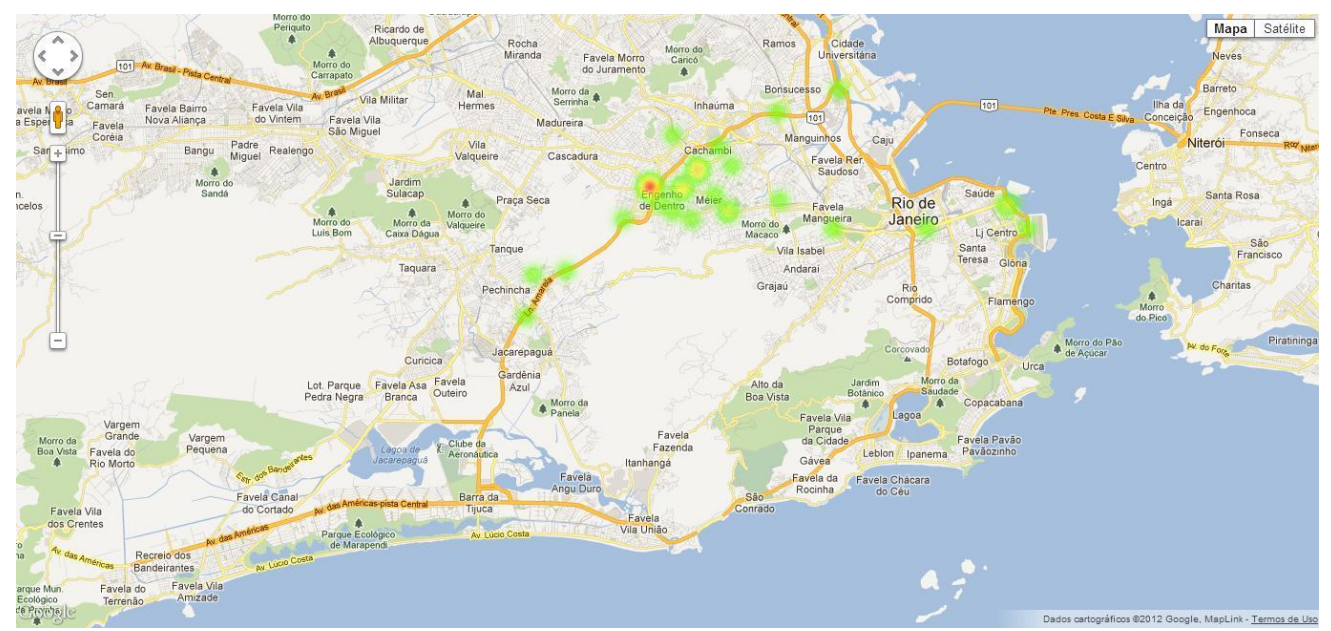

Figura 10. Mapa dia 30/10/11 - $t_{\text {departure }}$

\section{CONCLUSÃO E TRABALHOS FUTUROS}

Com a análise de alguns jogos do Campeonato Brasileiro de Futebol de 2011, foi possível identificar padrões de comportamento do usuário em relação às ligações realizadas durante os eventos. Além disso, através do processo de geração de mapas de calor, construído pelo aluno de iniciação científica, percebeu-se um aumento na utilização das antenas quando existe algum tipo de acontecimento na região. Com esses dados, é possível que as operadoras de serviço móvel estruturarem de forma melhor a rede e assim forneçam serviços de melhor qualidade, principalmente durante a próxima Copa do Mundo e Jogos Olímpicos que serão sediados no Brasil.

Outro fator percebido é que a quantidade de ligações pode sofrer alterações mesmo para eventos do mesmo tipo em dias diferentes. Esta diferença pode ser percebida durante os jogos de futebol dos dias 28/08 e 04/12 que apresentaram comportamentos distintos devido, principalmente, a fatos que aconteceram no dia 28/08 (AVC do técnico do Vasco). Com isso, as operadoras de telefonia móvel não só devem estruturar a sua rede de forma melhor, mas também devem levar em consideração fatores que podem interferir na quantidade de ligações. Assim, elas podem conseguir disponibilizar melhores serviços para cada tipo de evento.

Como trabalhos futuros, pode-se expandir a análise e a aplicação da metodologia para novas bases de dados e diferentes tipos de eventos, como shows, corridas, entre outros. Além disso, uma outra sugestão é a análise do envio de SMS e utilização da rede 3G/4G. Com isso, aplicar e, caso necessário, adaptar a metodologia proposta, comparando assim se o comportamento dos usuários é similar ou distinto em relação a diferentes tipos de eventos e tecnologias de comunicação.

Por último, a partir da análise, direcionar o desenvolvimento de aplicativos para aparelhos móveis e simuladores que ajudem na predição de carga na rede móvel da região; bem como na criação de ferramentas sobre geração e gerenciamento dos mapas de calor, que o usuário possa editar e visualizar da forma que achar melhor eventos anteriores e estimar eventos futuros. 


\section{REFERÊNCIAS}

Balcan, D. et al. Multiscale mobility networks and the spatial spreading of infectious diseases. Proceedings of the National Academy of Sciences, v. 106, n. 51, p. 2148421489, 2009. Disponível em: <http://www.pnas.org/content/106/51/21484.abstract>.

Bagrow, J. P.; Wang, D.; Barabási, A.-L. Collective response of human populations to large-scale emergencies. PLoS ONE, Public Library of Science, v. 6, n. 3, p. e17680, 032011.

Bleicher, A. The on-demand olympics. IEEE Spectrum, jul 2012.

Candia, J. et al. Uncovering individual and collective human dynamics from mobile phone records. Journal of Physics A: Mathematical and Theoretical, v. 41, n. 22, p. 224015, 2008. Disponível em: 〈http://stacks.iop.org/1751-8121/41/i=22/a=224015>.

Eagle, N.; Pentland, A.; Lazer, D. Inferring social network structure using mobile phone data. Proceedings of the National Academy of Sciences (PNAS), v. 106, n. 36, p. 15274-15278, 2009.

González, M. C.; Barabási, A.-L. Complex networks - from data to models. Nature Physics, v. 3, p. 224-225, 2007.

González, M. C.; Hidalgo, C. A.; Barabási, A.-L. Understanding individual human mobility patterns. Nature Publishing Group, v. 453, 2008. Disponível em:

<http://dx.doi.org/10.1038/nature06958>.

Mitchell, T. M. Mining our reality. Science, v. 326, n. 5960, p. 1644-1645, 2009. Disponível em: 〈http://www.sciencemag.org/content/326/5960/1644.short>.

Schopenhauer, A.Simonite, T. Mobile Data: A Gold Mine for Telcos. may 2010. Intelligent Community Forum.

Song, C. et al. Modelling the scaling properties of human mobility. Nature Publishing Group, v. 6, 2010. Disponível em: <http://dx.doi.org/10.1038/nphys1760>.

Song, C. et al. Limits of predictability in human mobility. Science, v. 327, n. 5968, p. 1018-1021, 2010. Disponível em:

<http://www.sciencemag.org/content/327/5968/1018.abstract>.

Soper, D. Is human mobility tracking a good idea? Communications of the ACM, ACM, New York, NY, USA, v. 55, n. 4, p. 35-37, abr. 2012. ISSN 0001-0782. Disponível em: <http://doi.acm.org/10.1145/2133806.2133819>.

Xavier, F. H. Z., Silveira, L. M., Almeida, J. M., Ziviani, A., Malab, C. H. S., e Marques-Neto, H. (2012). Analyzing the workload dynamics of a mobile phone network in large scale events. Em Proceedings ofthe UrbaNe Workshop - ACM CoNEXT 2012. 